\title{
Individuality of photoresponse dynamics of semiconductor sensors
}

\author{
V.P.Mygal, I.A.Klymenko, G.V.Mygal \\ National Aerospace University named by N.Zhukovsky "KhAI", \\ 17 Chkalova Str., 61070 Kharkiv, Ukraine
}

Received November 28, 2016

\begin{abstract}
Influence of the direction of external electric field on the energy spectrum of electronic states and dynamics of the processes of generation and recombination of charge carriers in crystals of zinc selenides was investigated. By geometrisation of dynamics of photoresponse in the parameter space it was revealed spatial and temporal correlation of their constituents. They determine the individual characteristics of the dynamics of the photoresponses.
\end{abstract}

Keywords: electronic states, zinc selenide, photoresponse.

Исследовано влияние направления внешнего электрического поля на энергетический спектр электронных состояний и динамику процессов генерации и рекомбинации носителей заряда в кристаллах селенида цинка. Посредством геометризации динамики фотооткликов в параметрическом пространстве выявлены пространственно-временные корреляции их составляющих. Они обусловливают индивидуальные особенности динамики фотооткликов.

Індивідуальність динаміки фотовідгуку напівпровідникових сенсорів. В.П.Мигаль, І.А.Клиленко, Г.В.Мигаль.

Досліджено вплив напрямку зовнішнього електричного поля на енергетичний спектр електронних станів і динаміку процесів генерації та рекомбінації носіїв заряду у кристалах селеніду цинку. За допомогою геометризації динаміки фотовідгуків у параметричному просторі виявлено просторово-часові кореляції їх складових. Вони обумовлюють індивідуальні особливості динаміки фотовідгуків.

\section{Introduction}

Operation of different nature sensors in extreme conditions (aerospace equipment, nuclear power plants, etc.) is characterized by a number of common problems for different sensors (dynamic compatibility of sensors in the matrix, variation of parameters, etc.) $[1,2]$. Thus, difficulty in selecting the same physical, biological and chemical sensors for redundancy is primarily related to the inability to detect the hidden dynamics of functional characteristics (FC) by traditional methods. Other indications of the dynamic individuality of sensors FC are their artifacts that reveal themselves mainly in extreme conditions. For semiconductor $\mathrm{A}_{2} \mathrm{~B}_{6}$ compounds and based on them sensors these problems are caused by influence of non- equilibrium growth conditions on formation of structural defects, which form in the piezoelectric crystal interconnected electrical and elastic fields. Influence of these fields is hidden in the subtle features of the temporal and spectral photoresponse (PhR) of the semiconductor sensor. Restructuring of the internal fields causes sensitized and non-additive photoconductive [3,4]. Obviously, the restructuring of the fields determines the character of the relationship between the FC. However, assuming that FC are one-dimensional temporal series of parameters, it is complicate to identify and analyze the hidden in them interconnectivity. However, the subtle structure of the spectral PhR semiconductor sensor depends 

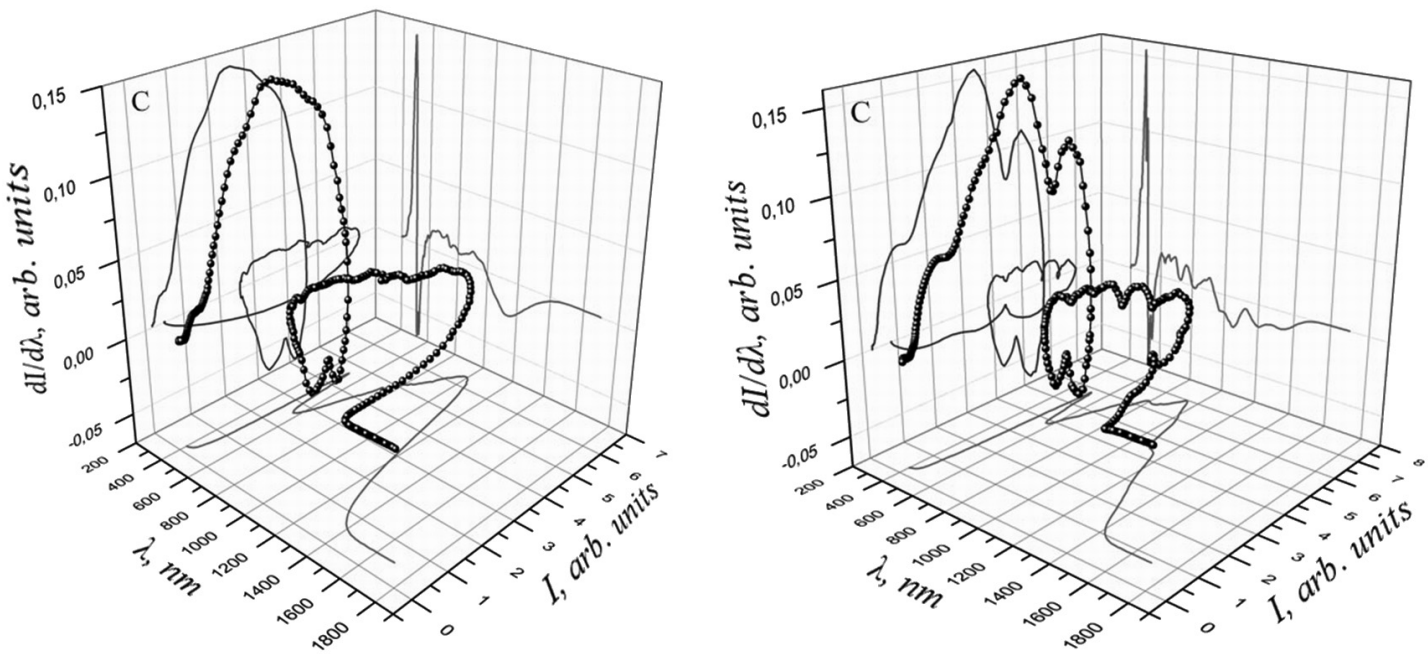

Fig. 1. Spectral PhR of ZnSe crystals, its derivatives and the 1st order signatures for the opposite direction of biasing field $E_{b}$.

on external factors (scanning time, frequency and form of bias field) [5,6]. Therefore, results of numerical differentiation of the spectral and temporal PhR are usually considered in the form of trajectory of dynamic states in the generalized phase space (state-velocity-time) [5]. The projection of the trajectory on the plane (state-velocity) is analyzed as a phase portrait. However, it consists of geometrically ordered sections. Individual sequence of the sections makes the portrait in the signature of the 1st order. Its configuration covers the area, which can be taken as a power of dynamic microstates subset, that allows to analyze statistically sensor PhR [7].

Additional numerical differentiation of the spectral and temporal PhR allows to extend the system analysis capabilities. The results of $\mathrm{PhR}$ measurements and differentiation can be represented in the space (state-velocity-acceleration) in the form of a closed trajectory of the dynamic events. Relations between the dynamical states and events is are hidden in the orthogonal projections of this trajectory, which is a signature of the 1st order, and two signatures of 2nd order. These relations can be investigated dynamically by comparing the configuration of the 1st and 2nd orders signatures, and also statistically - by analysis of areas ratio, that they cover in two or four quadrants [2]. This allows you to explore the individuality in the time and spectral sensor PhR, that is the main purpose of this article.

\section{Experimental}

ZnSe crystals were chosen as an object of our research. These crystals are widely used as sensors, detectors, scintillators and spectrometers. ZnSe crystals were grown from melt by the modified Bridgman method of vertical crystallization. The samples had the rectangular parallelepiped form with dimensions of $10 \times 10 \times 3 \mathrm{~mm}^{3}, 12 \times 6 \times 4 \mathrm{~mm}^{3}$. Their resistivity was $\rho \geq 10^{12} \mathrm{Ohm} \cdot \mathrm{cm}$. Structural research discovered defects of different types and sizes in the samples [8]. Their diversity and complexity of distribution determined the individual technology-inherited defect structure and complex energy spectrum [9]. The indium-gallium contacts were created on the greatest opposite sides of the samples. Measurements of spectrum $I(\lambda)$ of crystals and the PhR kinetic $I(t)$ were carried out under field strength $E \sim 3 \cdot 10^{2} \mathrm{~V} \cdot \mathrm{cm}^{-1}$ and a temperature of $293 \mathrm{~K}$. For separation of subtle dynamic features the $\mathrm{PhR} I(t)$ signal was digitized at a sampling rate $f_{d}>10^{3} \mathrm{~Hz}$ using data collection system ADVANTECH PCI1711L, followed by treatment with a computer. Processing of PhR and determination of the differential-geometric parameters of his signatures on the phase plane were implemented in the software package Octave.

Presentation of the results of ZnSe crystals spectral PhR scanning in the generalized phase space (velocity-state-time) transforms a one-dimensional set of parameters to a complex trajectory of photoinduced energy states of the crystal (Fig. 1, three-dimensional curve). It can be seen the differ- 


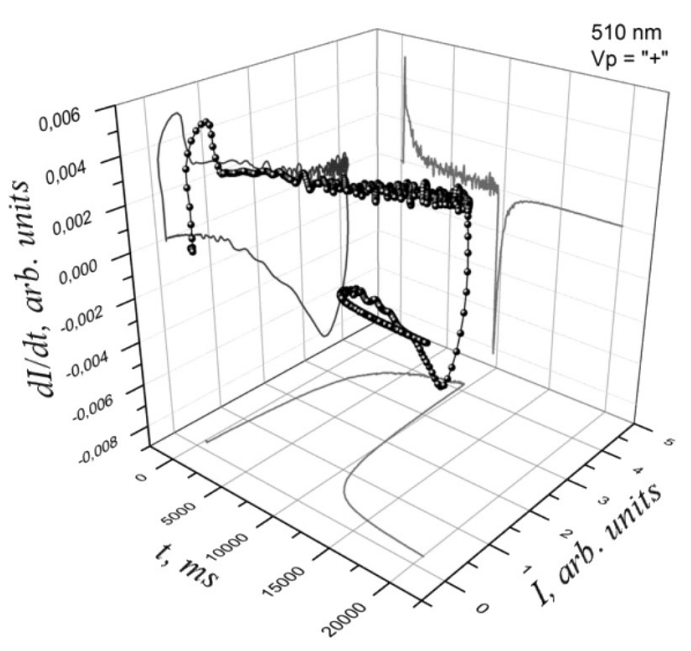

a)

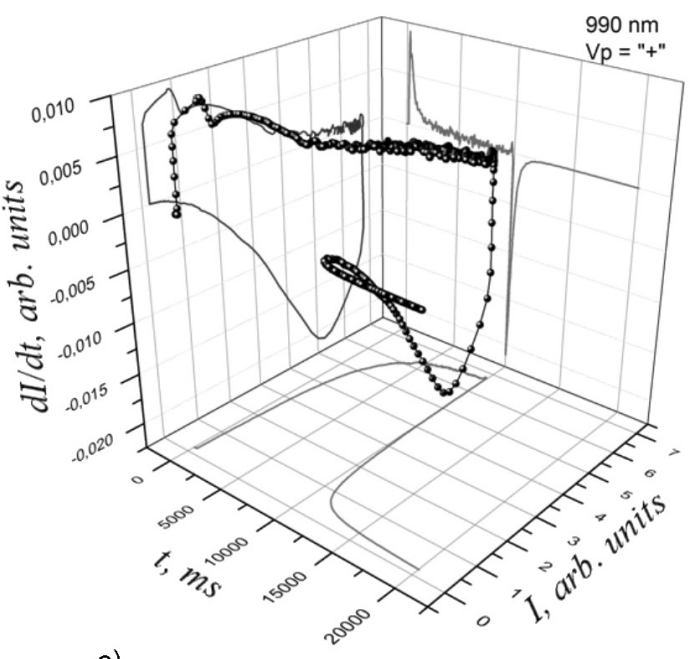

c)

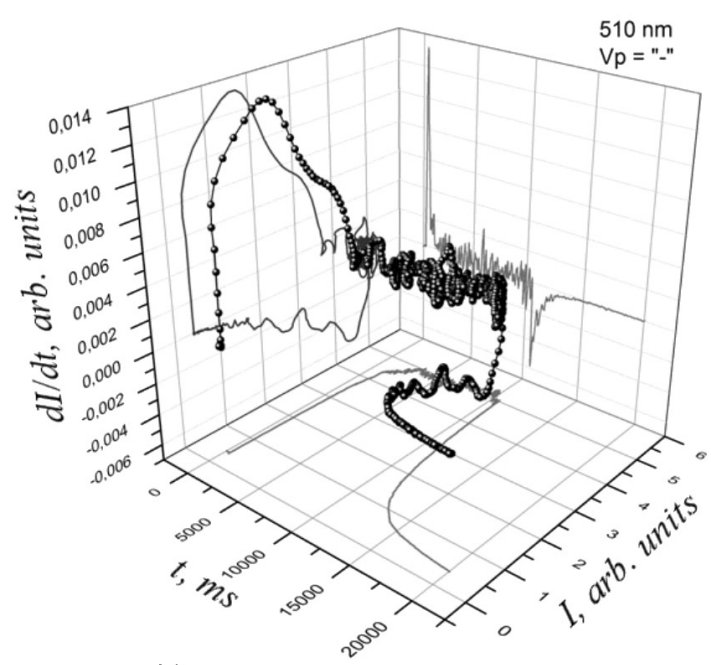

b)

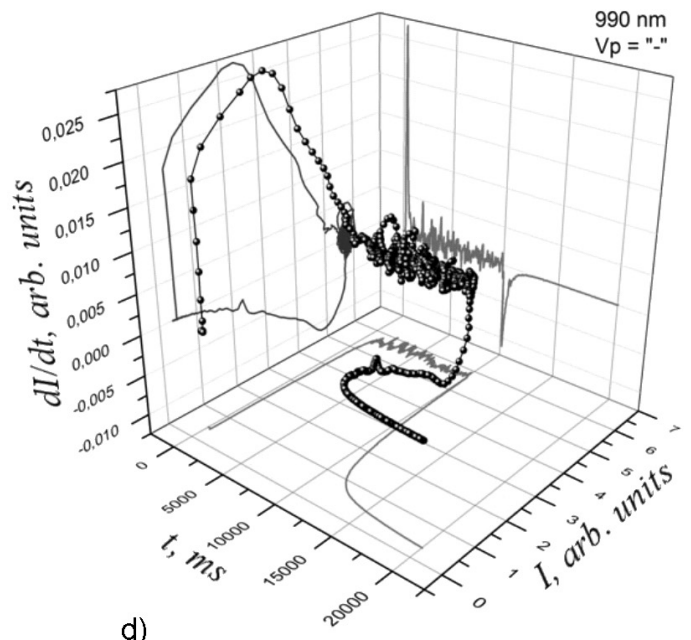

d)

Fig. 2. Kinetics $I(t)$ of ZnSe crystals PhR, their derivatives and signature of the 1st order for the opposite direction of biasing field.

ent spatial distribution of the sets of dynamic states that correspond to different bands of photosensitivity.

Individuality of the spectral $\mathrm{PhR}$ is clearly visible in the projections of trajectories onto the plane (velocity-state) (Fig. 1, plane $\mathrm{C}$ ). In this regard, the projection trajectories are signatures of the spectrum of the 1 st order $I(\lambda)-d I(\lambda) / d \lambda$. The difference between these signatures for opposite directions of biasing field $E_{b}$ is sufficiently informative. Thus, a comparison of the spectral PhR signatures configurations for the opposite directions $E_{b}$ help to resolve the spectrum bands with maxima of $570 \mathrm{~nm}$, $650 \mathrm{~nm}$ and $780 \mathrm{~nm}$, which are clearly appeared only providing one direction of the $E_{b}$. Other aspects of PhR structuring also clearly visible.
Kinetics of the photocurrent $I(t)$ provided the photoexcitation of $510 \mathrm{~nm}$ and $990 \mathrm{~nm}$, and the derivatives thereof are shown in Fig. 2. They are similar in form and for different directions $E_{b}$ differ fluctuations level. However, after converting them to the trajectories of dynamic states is visible PhR dynamics individuality and its dependence on the direction of $E_{b}$. It is most evident in the anisotropy of the signatures configurations and in difference of areas covered by them (see Fig. 2 a, c). The picture clearly shows the inversion of configuration of $\mathrm{PhR}$ kinetics signature, i.e. converting of antiphase kinetics components.

As expected, the presentation of the temporal PhR as a trajectory of dynamic events revealed individuality of the trajectories projections on the planes (state-acceleration) 


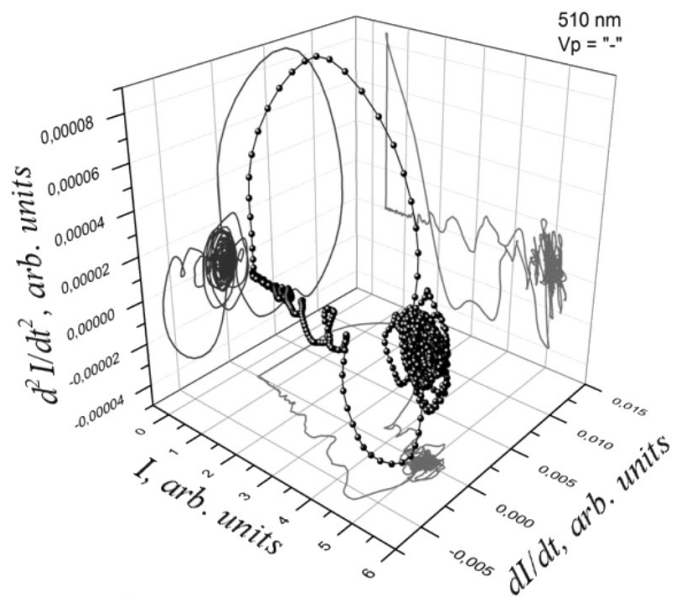

a)

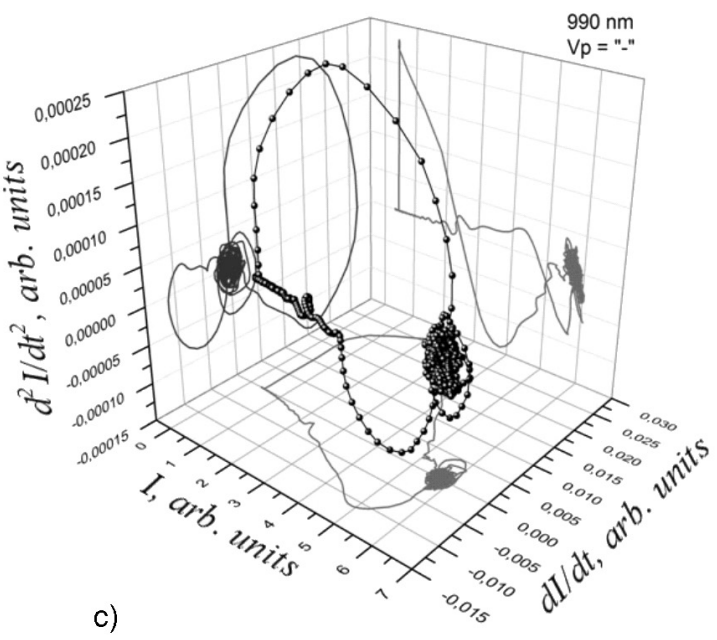

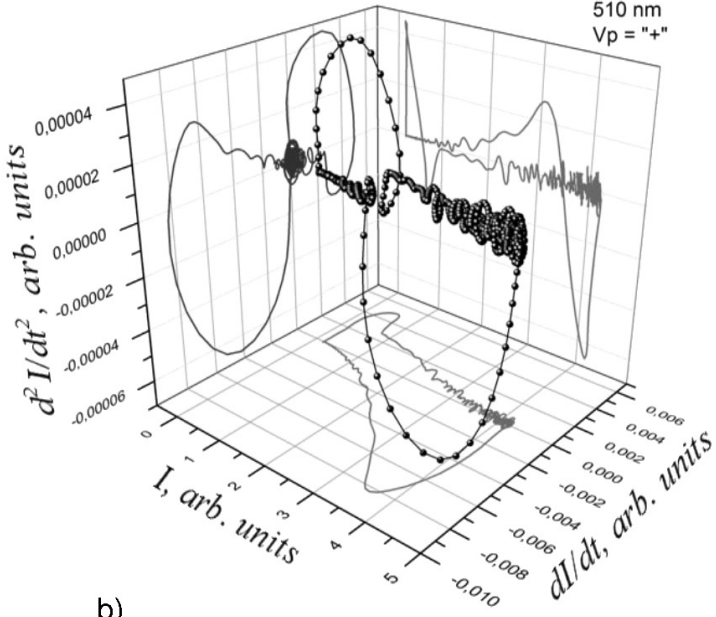

b)

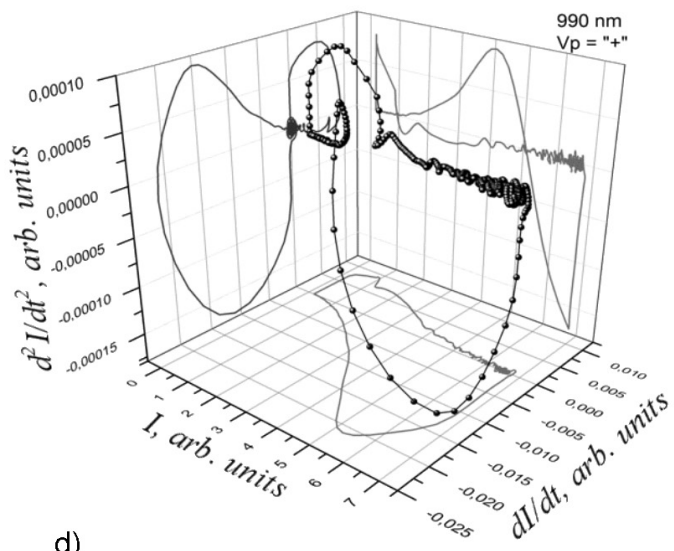

Fig. 3. The 1st and 2nd order signatures of ZnSe crystals transition $\mathrm{PhR}$.

and (velocity-acceleration), i.e. at signatures in the 2nd order (see Fig. 3). Thus, individual differences of signatures configurations of kinetics of the 2 nd order on the plane (state-acceleration) are appeared in the relationship of antiphase extremes areas. They show the influence of internal fields on the dynamics of PhR. The projection of the dynamic events trajectory on the plane (velocity-acceleration) is the 2nd order signature of PhR. Its configuration is determined by the nature of the relationship of dynamic variables in 4 quadrants which emphasize the basic phases of temporal $\mathrm{PhR}$. The change in direction of the external field is held simultaneously with the antiphase processes inversion.

As can be seen, the individuality of photovoltaic processes dynamics and the nature of influence on it of the energy spectrum of electronic states define the configuration of the sensor PhR signature.
Representation of the spectral PhR in the form of dynamic events trajectory revealed their significant difference for the opposite directions $E_{b}$. It is most evident in the projection of the trajectory on the planes (state-velocity), (state-acceleration) and (velocity-acceleration), i.e. in discussed above signature of the 1st order as well as in two signatures of the 2 nd order (see Fig. 4). The projection of the trajectory on the plane (state-acceleration) is a signature of the 2 nd order $I(\lambda)-d^{2} I(\lambda) / d \lambda^{2}$. In it the influence of $E_{b}$ direction appeared as the number of extremes and as the ratio of areas of the antiphase extremes. The projection of the trajectory of dynamic events on the plane (velocity-acceleration) is a signature of the 2 nd-order of spectrum. Its configuration consists of convex sections and loop-like sections the number of which depends on the direction of $E_{b}$, and on the time of the spectral PhR scanning. There- 

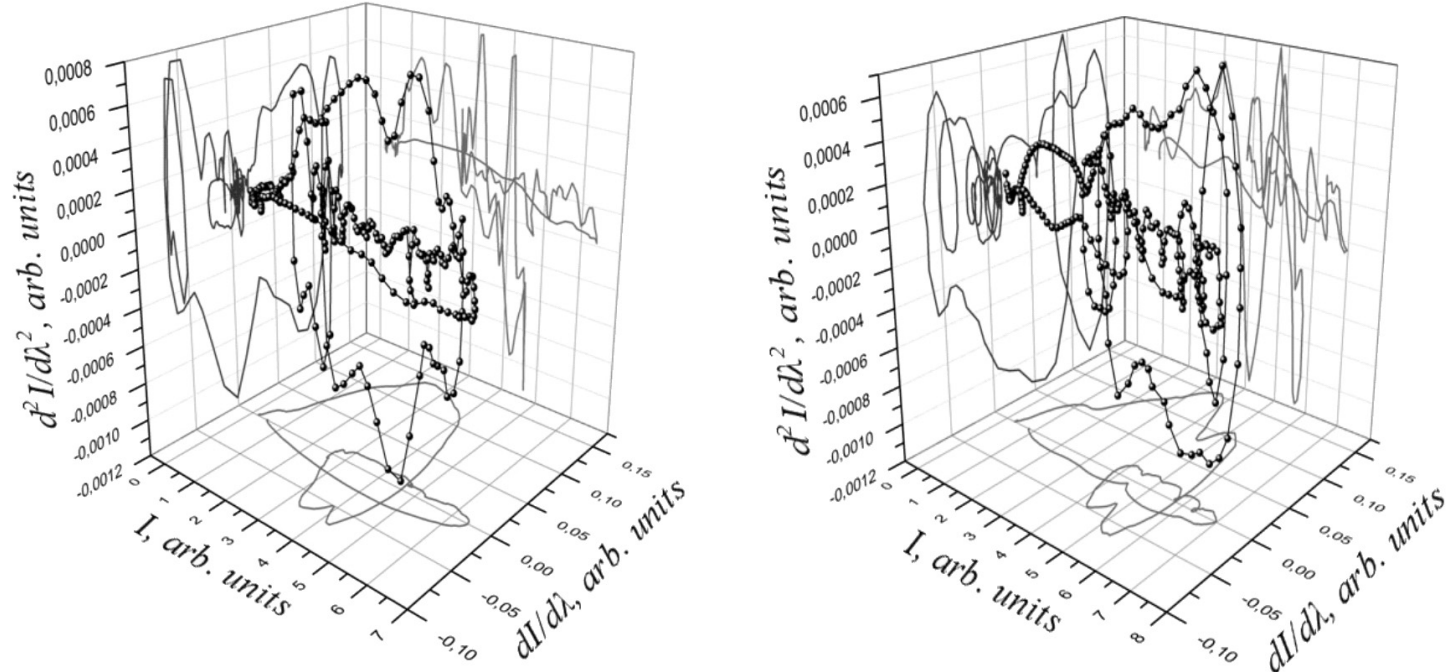

Fig. 4. The 1st and 2nd order signatures of ZnSe crystals spectral PhR.

fore, the signature configuration is defined not only dependence of $\mathrm{PhR}$ on the wavelength, but also on features of the temporary $\mathrm{PhR}$. Location of the signature configuration in 4 quadrants separates at space the main components of the spectral PhR, which form its structure. It determines the dynamics of generation-recombination processes and, therefore, very sensitive to changes in the direction of $E_{b}$.

Note, correlation of the manifold subtle features of the spectral PhR in the 1st and 2nd order signatures can detect as the elements of dynamic similarity, as well as the characteristic features of its structure (see Fig. 4).

\section{Results and discussion}

The manifold of defects and their distribution in compounds $A_{2} B_{6}$ forms an individual potential relief, which affects the energy spectrum of electronic states and dynamics of the generation-recombination processes [10]. It is obvious that their correlation leads to a hidden individuality of the dynamics of the sensors PhR. It appears in configuration of the 1 st and 2 nd order signatures of time and spectral PhR. Their informative value caused by three backbone factors. Firstly - natural decomposition of PhR on the geometrically-ordered sectionscomponents that differ events linear density, therefore, intervals between events. Secondly - the components differ by the universal differential-geometric parameters of slope or curvature, which correspond to the velocity or acceleration of the PhR. In the third configurations the $\mathrm{PhR}$ signatures can be analyzed holistically as a subset of photoinduced microstates. So the 1st order signature configuration covers the area, which can be represented as power $\left|W_{i}\right|$ of subset of the possible induced dynamic microstates, and the natural logarithm of $\left|W_{i}\right|$ is $\mathrm{PhR}$ entropy [11].

While the change in the direction of external field $E_{b}$ almost no effect on the spectral and temporal $\mathrm{PhR}$ of $\mathrm{ZnSe}$ crystal in usual form, their signatures differ significantly. Thus, the comparative analysis of the signatures of temporal $I(t)$ and spectral sensors $\mathrm{PhR}$ reveal that the change in $E_{b}$ direction causes inversion of configuration of the kinetics signatures sections, that display anti-phase processes. Inversion accompanied by the changes in attitudes of covered by signatures areas and, respectively, powers of the dynamic microstates subsets. In this case, the signatures of $\mathrm{PhR}$ kinetics of different spectral bands are similar, but differ subsets powers of photoinduced states $V_{1}$ and $V_{2}$. This, as well as changing the bands position in the spectrum signature, can be associated with the inversion of photocarriers sign when the IR photoexcitation, which was detected in these crystals [12]. Note, there is smaller area of the signature of $\mathrm{PhR}$ infrared band with a maximum at $990 \mathrm{~nm}$, which corresponds to the lower entropy. This can be related to identification of the ordering of two-dimensional structural defects in these crystals [13].

Overlap area of the bands at 510 and $990 \mathrm{~nm}$ in the spectral PhR signature can be represented as the power of subset of general induced microstates, i.e. it is the intersection of subsets $V_{1} \cap V_{2}$, displaying 
these bands. The ratio of subsets powers of general $V_{1} \cap V_{2}$ and individual $V_{1} \cap V_{2}$ microstates characterizes the relationship between respective photosensitivity centers. It displays an integrative index $W_{1}=\left(V_{1} \cap V_{2}\right)$ $R\left(V_{1} \cap V_{2}\right)$. Note also, that the sensors with less power of induced microstates subsets have indicator of the dynamic balance $B_{d y n}$ of antiphase processes in $\mathrm{PhR}$ close to one. Indicator of the dynamic balance $B_{d y n}$ is equal to the ratio of area of upper $S_{u}$ and lower $S_{l}$ parts of the signatures $B_{d y n}=$ $S_{u} / S_{l}$ [2]. Accumulated statistics confirms that operation of the sensors in extreme conditions decreases dynamic balance, that is often accompanied by fluctuations, the appearance of instability and artifacts. Therefore, the transition from a time series of parameters of different $\mathrm{PhR}$ to the trajectory of dynamic states allowed to expand the analysis of the signal dynamics by statistics consideration.

Converting of $\mathrm{PhR}$ to the closed trajectory of dynamic event of space (state-velocity-acceleration) provides new opportunities. Firstly, the orthogonal projections of the trajectory are configurations of the 1st and 2nd order signatures, which allowed to analyze the temporal and spectral $\mathrm{PhR}$ from three points of view - dynamic, energetic and structural-functional, which provide a coherent information. Secondly - the signatures configurations of different $\mathrm{PhR}$ indicate its natural decomposition into components having different physical meaning, but they are characterized by the same differential-geometric parameters (slope, curvature). In the third - the signatures areas can be considered as the power of photoinduced microstates subset. It is made possible to supplement the dynamic analysis of PhR by the statistical analysis. In addition, in the analysis of $\mathrm{PhR}$ can be used universal parameters (entropy), as well as integrative indicators of dynamic ordering of antiphase processes and their energy balance [2].

The comparative analysis of the signatures of the 1 st and 2 nd order of the spectral and temporal $I(t) \mathrm{PhR}$ of ZnSe crystals upon excitation of the impurity band maxima photosensitivity (maxima of $510 \mathrm{~nm}$ and $990 \mathrm{~nm}$ (see Fig. 1)) is made. It shows that the impurity photosensitivity centers generating spectral bands with maxima at $510 \mathrm{~nm}$ and $990 \mathrm{~nm}$ have different dynamics and nature. Overlap of configurations signatures of the 1st order spectral bands indicates the presence of a subset of the overall photoinduced microstates. The ratio of subsets powers of the common and individual microstates is an indicator of the relationship of the respective photosensitivity centers. Furthermore, joint analysis of the examined signatures of the spectral and temporal sensor PhR shows the impact on the dynamics of the processes of charge carriers generation and recombination of the internal field self-consistent restructuring. Therefore, selection of the same sensors for spectral PhR signatures was the most effective. Similar form of structure of the $\mathrm{PhR}$ of different nature sensors indicates the similarity of their dynamic processes. It can be assumed that the operation of such sensors is dynamically consistent. This is confirmed by the fact that ZnSe crystals, using as photodetectors, and also as scintillator [14], with similar structures of the PhR spectra and X-ray spectra give a good interoperability. Despite the different nature of the FC configurations of their 2nd order signatures were similar [15]. Therefore, their operation structures are also similar that allowed to offer an efficient way of radiation detection.

Note, that relationship of the dynamics and structure of the sensor response follow from several fundamental principles. Thus, the principle of mechanical determinism defines the causal relationships in a sequence of the dynamic sensor states. The principle of structural determination binds the ordering of structure of management system and its function [16]. In the nature of the self-consistent restructuring of elastic and electric fields of the crystal for maximum resistance to external factors shows the Le Chatelier-Braun principle. Therefore it can be assumed that these principles define the interrelated dynamic and energy aspects of the signals structure. This, in particular, is confirmed by the inherited technologically dynamic individuality of the semiconductor sensors response [17]. It is caused by the structural defects and residual stresses whose influence is the most evident in the 1 st and 2 nd order signature of the spectral and temporal $\mathrm{PhR}$ of ZnSe crystals.

\section{Conclusions}

Thus, transformation of the various functional characteristics of the sensors in the 1st and 2nd order signatures extends the capabilities of the system analysis of the complex research results. In particular, a sufficiently high sensitivity of the differential-geometry indicators and integral indi- 
cators of the sensor PhR signatures to internal and external factors has the high prospects for their use for monitoring at all stages of the sensor life cycle. The relationship of the dynamics and structure of the sensor response is interesting for study and simulate the dynamic behavior of different types sensors, including chemical and biological, under extreme conditions.

The article contains the results of studies conducted with grant support from the $\mathrm{Na}$ tional Foundation for Fundamental Research on the contest project F64-5862.

\section{References}

1. Y.Lepikh, et al., The Design of New Generation Microelectronic Sensors for Intelligent Systems. - Odesa, Ukraine: Astroprint., 2010. 296 p. (Ukraine)

2. V.P.Mygal, A.V.But, G.V.Mygal, I.A.Klimenko, Sci Rep., 6, 29512, (2016).

3. I.G.Gavrikova, V.P.Migal, A.L.Rvachev, Phys. Stat.Sol.(A), 37, K21 (1976).

4. V.P.Migal, Semicond., 35, 1135 (2001).

5. A.V.But, V.P.Mygal, A.S.Phomin, Semicond., 43, 581 (2009)
6. V.K.Komar, V.P.Migal, O.N.Chugai et al., Appl. Phys. Lett. 81. 4195 (2002).

7. But A.V., Mygal V.P., Bodnar I.V., Opt. Syst. Des. Proc. SPIE 8550, 85502B (2012).

8. A.V.But, V.P.Mygal, I.V.Bodnar, Opt. Syst. Des. (2012).

9. O.N. Chugai, A.S. Gerasimenko, V.K. Komar et al., Phys. Solid State, 52, 2467 (2010).

10. V. P. Mygal, A. V. But, A. S. Phomin, I. A. Klimenko, Semicond., . 49, 634 (2015).

11. A.Keith, Entropy. Amer. J. Phys., 52, 492 (1984). http://dx.doi.org/10.1119/1.13892

12. Gavrikova I.G., Migal V.P., Rvachev A.L., Phys. Stat. Sol.(a), 30, K95-K97 (1975).

13. O.N. Chugai, S.L. Abashin, A.V. Gaidachuk et al., Inorg. Mat., 51, 972 (2015).

14.L.V.Atroshchenko , L.P. Gal'chinetskii , S.N. Galkin, et al., J. Cryst. Growth, 198/199, 292 (1999).

15. V.P.Mygal, G.V. Mygal, Electrotechn. Comp. Syst., 22(98), 354 (2016).

16. V.I. Knorring . Theory, Practice and Art of Management. Norma, Moscow (2001) [in Russian], p.321.

17. V.P. Mygal, A.V. But, G.V. Migal, I.A. Klymenko, Funct. Mater., 22 (3), 387 (2015). 